\title{
Efficacy of Unsupervised Home-Based Pulmonary Rehabilitation for Patients with Chronic Obstructive Pulmonary Disease
}

This article was published in the following Dove Press journal: International Journal of Chronic Obstructive Pulmonary Disease

\author{
Jang Ho Lee (1) \\ Hyang Yi Lee \\ Youngwon Jang \\ Jae Seung Lee (D) \\ Yeon-Mok Oh (iD) \\ Sang-Do Lee \\ Sei Won Lee (ID
}

Department of Pulmonary and Critical Care Medicine, Asan Medical Center, University of Ulsan College of Medicine, Seoul, Republic of Korea
Correspondence: Sei Won Lee Department of Pulmonary and Critical Care Medicine, Asan Medical Center, University of Ulsan College of Medicine, Songpa-Gu, Seoul 05505, Republic of Korea

Email iseiwon@gmail.com
Purpose: Pulmonary rehabilitation (PR) is a well-established treatment for chronic obstructive pulmonary disease (COPD). The standard protocol for PR requires frequent hospital visits, which can be difficult for patients. We performed this study to assess whether unsupervised home-based PR (HBPR) is effective for patients with COPD.

Patients and Methods: After assessing the outcome data, including the results of a COPD assessment test (CAT); the body mass index, airflow obstruction, dyspnea, and exercise capacity (BODE) index; a spirometry; the modified Medical Research Council (mMRC) dyspnea scale; and the 6-min walking test (6MWT), specialists imparted 1-hour education to patients regarding unsupervised HBPR at the baseline visit. This included methods for breathing, inhaler use, stretching, and exercise. On reviewing diaries after 8 weeks from the first visit, patients who exercised at least thrice per week were classified as the compliant group and the others were categorized as the noncompliant group. Changes in the outcomes were compared between the compliant and noncompliant groups.

Results: A total of 41 patients were enrolled in this study; for 8 weeks of unsupervised HBPR, there were significant improvements in CAT scores ( $-4.62 \pm 4.61$ vs $2.40 \pm 6.73$; $P=0.002)$, BODE index $(-1.00 \pm 1.06$ vs $-0.20 \pm 0.56 ; P=0.01)$, and forced expiratory volume in $1 \mathrm{~s}(0.05 \pm 0.19$ vs $-0.09 \pm 0.16 ; P=0.02)$ among patients in the compliant group, compared with the noncompliant group. Moreover, their CAT $(16.46 \pm 7.80$ vs $11.85 \pm 7.23 ; P=0.03)$ and mMRC scores $(2.54 \pm 0.76$ vs $1.81 \pm 0.63 ; P=0.001)$ improved significantly after 8 weeks, compared with those at baseline. On the other hand, patients in the noncompliant group showed no significant improvement in any of the outcomes.

Conclusion: In this study, compliant patients with unsupervised HBPR achieved favorable outcomes in 8 weeks. Thus, we recommend unsupervised HBPR for patients with COPD, even when regular hospital visits for PR are not possible.

Trial Registration: NCT03754881.

Keywords: chronic obstructive lung disease, chronic obstructive lung disease assessment test, home-based pulmonary rehabilitation, modified Medical Research Council, pulmonary rehabilitation

\section{Introduction}

Chronic obstructive pulmonary disease (COPD) is the fourth leading cause of death worldwide, with a global prevalence of $11.7 \% .{ }^{1}$ Currently, inhaled bronchodilators are the mainstay of treatment for COPD. Although bronchodilators can improve lung function, as well as alleviate symptoms and reduce acute exacerbation in patients with $\mathrm{COPD},{ }^{2-4}$ evidence regarding their effect on these patients' 
performance and exercise capacity remains relatively insufficient. Because of this limitation associated with COPD pharmacotherapy, there is the need for a combination of nonpharmacologic therapies, which should include smoking cessation, pneumococcal vaccinations such as PCV13 and PPSV23, pulmonary rehabilitation (PR), and long-term oxygen supply. Among these therapies, PR has been considered as one of the most wellestablished treatments for COPD. ${ }^{5}$

PR constitutes a multidisciplinary intervention that includes exercise training, physical therapy, and patients education. ${ }^{6}$ Although PR can be applied to several chronic respiratory diseases, patients with COPD are known to benefit most from PR. Studies have shown that PR improves exercise capacity, dyspnea, quality of life and reduces acute exacerbations, hospital admission, along with depression in patients with COPD. ${ }^{5,7-12}$ In addition, landmark studies on PR have included programs with hospital visits of 2 or 3 days per week for a period 8-12 weeks, ${ }^{13,14}$ with each session consisting of 1-4 h of PR training under supervision. Although this standard protocol is reasonable, it is not easy to perform in practice due to the frequent hospital visits required. ${ }^{15}$ Hence, to overcome this issue, several evidence-based studies have suggested home-based PR (HBPR). ${ }^{16-21}$ Nevertheless, the majority of these studies were performed with relatively frequent periodic supervision of specialists, which was not always feasible in communities with limited medical resources. Owing to these limitations, compliance with PR remains inadequate among patients.

Thus, to apply PR to patients with COPD and enable them to understand its concept, it might be performed intermittently at patients' clinic visits every 2-3 months. However, there is a lack of firm evidence supporting this practice. Therefore, in the present study, we educated patients with COPD at baseline about HBPR and trained them to perform it without supervision. After 8 weeks, we compared the compliant group with the noncompliant group in terms of the improvement in the quality of life, dyspnea, spirometry, and 6-min walking test (6MWT) results, along with other prognostic factors. We aimed to determine the feasibility and the preliminary efficacy of an unsupervised HBPR in patients with COPD. In this study, we sought to validate the superiority of outcomes among the compliant patient group versus the noncompliant group in the 8 weeks of unsupervised HBPR.

\section{Patients and Methods}

\section{Patients and Study Design}

We conducted a prospective cohort study with an enrollment of a total of 41 COPD patients at the Asan Medical Center in South Korea. Eligible patients with COPD were screened, considering the following inclusion criteria: patients (1) with forced expiratory volume in $1 \mathrm{~s}\left(\mathrm{FEV}_{1}\right)$ /forced vital capacity (FVC) $<0.7$; (2) aged $>40$ years; (3) with no history of acute exacerbations within the past 4 weeks; (4) with no history of PR within the past 6 months; (5) with no change in the COPD maintenance regimen within the past 3 months; and (6) with modified Medical Research Council (mMRC) dyspnea scale score $>1$. Our exclusion criteria were (1) patients with the presence of comorbidity that made PR difficult, including cardiac disease, orthopedic disease, neurologic disease, visual disturbance, or uncontrolled hypertension and (2) patients under long-term oxygen therapy.

After the assessment of spirometry and 6MWT results, responses to questionnaires of COPD assessment test (CAT) scores $^{22}$ and dyspnea at baseline, patients underwent HBPR that included supervised exercise, education, and physical therapy for $1 \mathrm{~h}$. Subsequently, clinicians recommended patients to perform unsupervised HBPR more than 30 mins per day and 3 times per week. Patients were provided with a booklet describing the prescribed exercise protocol and a diary to record information about their exercise habits (Supplementary Figure 1). For 8 weeks period, we made weekly calls to examine and encourage the patients and verify whether they were performing HBPR and maintaining an exercise diary without visiting hospitals. After 8 weeks of HBPR, patients again underwent spirometry, 6MWT, questionnaire, and CAT for reevaluation. We then reviewed their diaries and categorized the patients into two groups. Those who exercised more than 30 mins per day and at least three times per week were classified as the compliant group, and the others were categorized as the noncompliant group (Figure 1). ${ }^{20}$

This study was approved by the Asan Medical Center's Institutional Review Board (No. 2018-0964) and registered at ClinicalTrials.gov (NCT03754881) in November 2018. Written informed consent was obtained from all study participants, and the study was conducted in accordance with the amended Declaration of Helsinki.

\section{PR Program}

The PR program included the following sessions: education about the methods for effective breathing and inhaler 


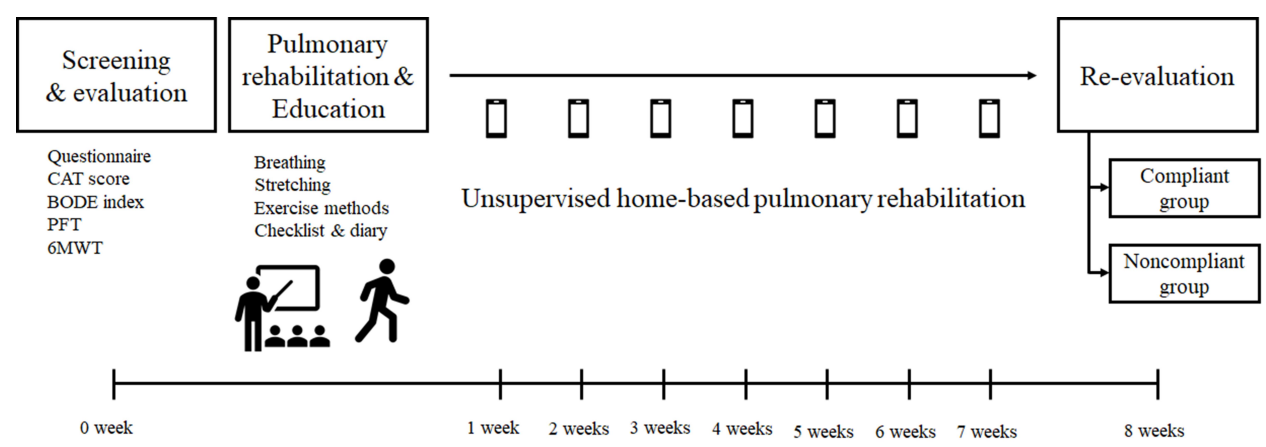

Figure I Study design for unsupervised home-based pulmonary rehabilitation. Baseline measurements were obtained after enrolling eligible patients. The patients performed unsupervised home-based pulmonary rehabilitation after receiving I-h education on it at baseline. The patients were called weekly and encouraged to achieve home-based pulmonary rehabilitation and maintain diaries of recordings. After 8 weeks, patients were categorized as either the compliant or noncompliant group, and measurements were analyzed for outcomes.

use, stretching, aerobic exercise, and muscle training for lower and upper extremities. For effective breathing, patients practiced abdominal breathing and pursed-lip breathing, inhaling through the nose and slowly exhaling with the lips closed. Clinicians and specialists evaluated the intensity of exercise that each patient should endure, according to his or her 6MWT results and endurance during the education sessions. ${ }^{23,24}$ Aerobic exercise consisted of walking or indoor cycling. Clinicians prescribed at least $20 \mathrm{~min}$ of aerobic exercise at $80 \%$ average 6MWT speed. $^{24}$ If it was challenging to estimate velocity during unsupervised HBPR, patients were recommended to exercise with a dyspnea level of 5-6 on a 10-point modified Borg scale. $^{25}$ Clinicians evaluated muscle strength during education sessions and prescribed intensity of $60-70 \%$ of maximal muscle strength. Rubber bands and weighted materials, such as sandbags, were used for muscle exercise. If it was difficult to maintain intensity, patients were encouraged to perform PR lower than the prescribed intensity. Clinicians recommended patients to exercise at least three times per week during the PR program.

\section{Data Collection and Measurement and Statistical Analysis}

All patients' data were collected from the electronic medical records and examined for baseline characteristics, medical history regarding COPD, past medical history, patient questionnaire responses, and laboratory test results. The primary outcome was change in the CAT score at 8 weeks. CAT consisted of questions concerning respiratory symptoms and quality of life. ${ }^{22}$ The secondary outcome was change in the mMRC score; the body mass index, airflow obstruction, dyspnea, and exercise capacity index (BODE index); $\mathrm{FEV}_{1}$; and 6MWT results at 8 weeks. The BODE index consisted of the following variables: $\mathrm{FEV}_{1}, 6 \mathrm{MWT}$, mMRC score, and body mass index. $^{26}$

We assumed that the probability of improvement in CAT with HBPR was approximately $70 \%$ in the compliant group, while the noncompliant group did not exhibit any improvement in CAT. Owing to the consideration of other factors, including inhaler use and other exercises, which could also affect patients with COPD, we assumed that the improvement in CAT of the noncompliance group was approximately $20 \%$. In our hospital, compliance with HBPR in outpatients was approximately $20 \%$. Our calculation demonstrated that 9 patients in the compliant group, and 36 patients in the noncompliant group were required to detect a significant difference at the 0.05 level $(p \leq 0.05)$ with a power of 0.8. We used MedCalc (Version 19.4.1) to calculate the sample size. We enrolled a total of 50 patients considering loss to follow-up.

Categorical variables were expressed as numbers and percentages of the participants. The $\chi^{2}$ or Fisher's exact test was used to analyze between-group differences. Continuous variables were presented as mean values with standard deviations. The Kolmogorov-Smirnov test was used to confirm the normality of distribution. Differences in continuous variables were analyzed using the Student's $t$-test, except for mMRC. Since the variables did not satisfy normality, the Mann-Whitney test was used to analyze the differences in $\mathrm{mMRC}$. All tests of significance were analyzed using two-sided tests; $P<0.05$ were considered to be statistically significant. All analyses were performed using the SPSS software (version 24.0; Chicago, IL, USA). 


\section{Results}

\section{Patient Enrollment and Baseline} Characteristics

During the study period, 50 patients with COPD were screened of whom 9 were excluded, including 6 due to loss to follow-up loss, 2 who had not undergone examination at 8 weeks, and 1 due to acute exacerbations during the study period (Figure 2). Table 1 shows the baseline characteristics of the remaining 41 participants, according to the study group. There were no significant betweengroup differences in the demographic and clinical characteristics between compliant and noncompliant groups such as age (68.69 \pm 9.34 vs $68.80 \pm 6.46, \mathrm{P}=0.969)$, sex, (male $96.2 \%$ vs $80.0 \%, \mathrm{P}=0.130$ ), BMI (22.96 \pm 3.26 vs $22.72 \pm 2.83, \mathrm{P}=0.809$ ), and smoking history (ever smoker $96.2 \%$ vs $73.3 \% \mathrm{P}=0.089$ ). The mean number of exercise sessions per week was $5.14 \pm 1.54$ in the compliant group and $1.18 \pm 1.59$ in the noncompliant group.

\section{Between Group Differences in Study Outcomes at 8 Weeks}

Table 2 presents the changes from baseline after 8 weeks in the two study groups. Clinical outcomes were significantly improved in the compliant group, compared with those in the noncompliant group in terms of the CAT score $(-4.62 \pm 4.61$ vs $2.40 \pm 6.73 ; P=0.002)$, BODE index $(-1.00 \pm 1.06$ vs $-0.20 \pm 0.56 ; P=0.01)$, and $\mathrm{FEV}_{1}(0.05$ \pm 0.19 vs $-0.09 \pm 0.16 ; P=0.02)$. The mMRC score $(-0.73 \pm 0.83$ vs $-0.27 \pm 0.88 ; P=0.183)$ and $6 \mathrm{MWT}$

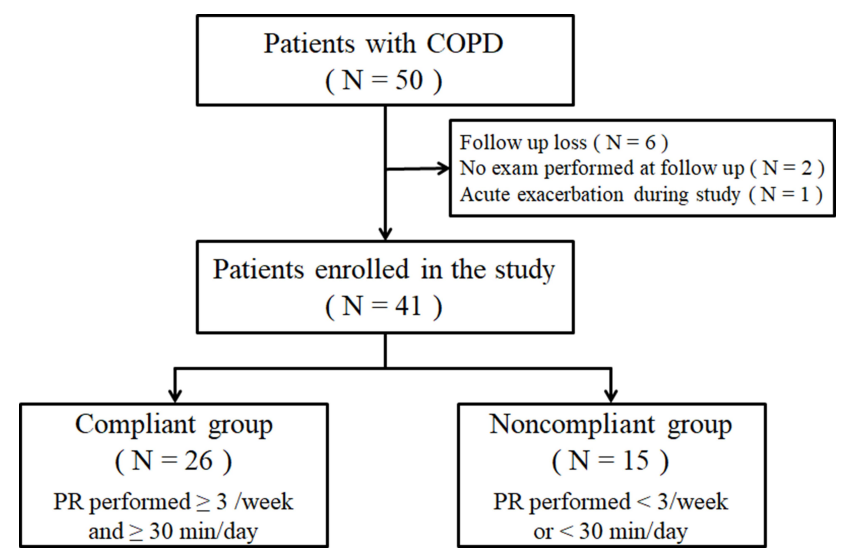

Figure 2 Study flow chart of the enrollment of patients with COPD for the unsupervised home-based pulmonary rehabilitation study. A total of 50 patients were screened for the study; of them, 9 patients were excluded. Among the enrolled $4 \mathrm{I}$ patients, 26 patients were grouped as compliant and 15 as noncompliant, based on adherence and nonadherence to 30 -min exercise per day for at least 3 days per week, respectively. results $(18.23 \pm 43.96$ vs $4.87 \pm 46.25 ; P=0.36)$ were also improved in the compliant group, but the differences were not significant.

\section{Within Group Differences in Study Outcomes at 8 Weeks}

The changes in baseline measurements of the two groups estimated after 8 weeks of PR are presented in Figures 3 and 4. There were significant improvements in the CAT $(16.46 \pm$ 7.80 vs $11.85 \pm 7.23 ; P=0.03)$ and $\mathrm{mMRC}(2.54 \pm 0.76 \mathrm{vs}$ $1.81 \pm 0.63 ; P=0.001)$ scores in the compliant group. However, the BODE index $(4.04 \pm 1.93$ vs $3.04 \pm 1.97$; $P=0.07), \mathrm{FEV}_{1}(1.32 \pm 0.47$ vs $1.37 \pm 0.46 ; P=0.71)$, and 6 MWT results $(400.35 \pm 97.31$ vs $418.58 \pm 88.07 ; P=0.48)$ presented no significant changes. In the noncompliant group, there were improvements in the mMRC score, BODE index, and 6MWT results, and the CAT score and $\mathrm{FEV}_{1}$ were also increased after 8 weeks; however, none of these changes exhibited a significant difference between baseline and after 8 weeks.

\section{Discussion}

This study demonstrated that unsupervised HBPR in outpatient clinics could indeed improve clinical outcomes in patients with COPD, thereby, suggesting that PR could be recommended even when supervised outpatient model requiring regular hospital visits are difficult. After 8 weeks of unsupervised HBPR, patients in the compliant group showed favorable outcomes; the CAT score, BODE index, and $\mathrm{FEV}_{1}$ were improved significantly. To our knowledge, this is the first study to suggest the benefits of unsupervised HBPR in compliant patients.

Also, the CAT and mMRC scores improved significantly by comparing the baseline estimates with those obtained after 8 weeks in the compliant group. Considering that a score of $\geq 2$ in CAT was regarded as a significant change,${ }^{27}$ the change of -4.61 in the present study indicated clinically meaningful improvement. ${ }^{28}$ The BODE index, one of the secondary outcomes in this study, is a wellknown prognostic index for patients with COPD. ${ }^{26,29}$ The compliant group showed a marginally significant improvement in the BODE index (from 4.04 to 3.04; $P=0.07$ ), which suggested that this type of unsupervised HBPR improved prognosis. Because only 41 patients were enrolled in this study, further investigation with more patients is necessary to prove an association between unsupervised HBPR and improved prognosis score, including 
Table I Baseline Characteristics

\begin{tabular}{|c|c|c|c|c|}
\hline & $\begin{array}{l}\text { Total } \\
(N=4 I)\end{array}$ & $\begin{array}{l}\text { Compliant Group } \\
(\mathrm{N}=\mathbf{2 6})\end{array}$ & $\begin{array}{l}\text { Noncompliant Group } \\
(N=15)\end{array}$ & $P$ value \\
\hline Male, n (\%)* & $37(90.2)$ & $25(96.2)$ & $12(80.0)$ & 0.130 \\
\hline Age (years) & $68.73 \pm 8.31$ & $68.69 \pm 9.34$ & $68.80 \pm 6.46$ & 0.969 \\
\hline $\mathrm{BMI}\left(\mathrm{kg} / \mathrm{m}^{2}\right)$ & $22.87 \pm 3.07$ & $22.96 \pm 3.26$ & $22.72 \pm 2.83$ & 0.809 \\
\hline Smoking, n (\%) & & & & 0.089 \\
\hline Current & $8(19.5)$ & $5(19.2)$ & $3(20.0)$ & \\
\hline Ex-smoker & $28(68.3)$ & $20(76.9)$ & $8(53.3)$ & \\
\hline Never smoker & $5(12.2)$ & I (3.8) & $4(26.7)$ & \\
\hline Smoking (pack*years) & $45.16 \pm 28.92$ & $50.75 \pm 29.35$ & $35.47 \pm 26.32$ & 0.104 \\
\hline \multicolumn{5}{|l|}{ Comorbidity, n (\%) } \\
\hline $\mathrm{DM}^{*}$ & $4(9.8)$ & $3(11.5)$ & I (6.7) & $>0.999$ \\
\hline HTN* & $9(22.0)$ & $3(11.5)$ & $6(40.0)$ & 0.053 \\
\hline Cardiovascular* & $5(12.2)$ & $2(7.7)$ & $3(20.0)$ & 0.336 \\
\hline Malignancy* & $8(19.5)$ & $5(19.2)$ & $3(20.0)$ & $>0.999$ \\
\hline History of acute exacerbation, $\mathrm{n}$ (\%) & & & & 0.735 \\
\hline 0 & $37(90.2)$ & $23(88.5)$ & $14(93.3)$ & \\
\hline I & $3(7.3)$ & $2(7.7)$ & I (6.7) & \\
\hline 2 & I (2.4) & I (3.8) & $0(0.0)$ & \\
\hline Drug compliance* & $37(90.2)$ & $24(92.3)$ & $13(86.7)$ & 0.615 \\
\hline Pre CAT & $16.49 \pm 7.56$ & $16.46 \pm 7.80$ & $16.53 \pm 7.38$ & 0.977 \\
\hline Pre mMRC, $\mathrm{n}(\%)$ & & & & 0.367 \\
\hline 2 & $28(68.3)$ & $16(61.5)$ & $12(80.0)$ & \\
\hline 3 & $7(17.1)$ & $6(23.1)$ & I (6.7) & \\
\hline 4 & $6(14.6)$ & $4(15.4)$ & $2(\mid 3.3)$ & \\
\hline Pre BODE index & $3.68 \pm 1.97$ & $4.04 \pm 1.93$ & $3.07 \pm 1.94$ & 0.129 \\
\hline \multicolumn{5}{|l|}{ Pre Spirometry } \\
\hline $\mathrm{FEV}_{\mathrm{I}}(\mathrm{L})$ & $1.35 \pm 0.51$ & $1.32 \pm 0.47$ & $1.41 \pm 0.58$ & 0.595 \\
\hline $\mathrm{FEV}_{1 \%}$ predicted & $47.80 \pm 16.69$ & $46.15 \pm 15.34$ & $50.67 \pm 19.01$ & $0.41 \mathrm{I}$ \\
\hline FVC (L) & $3.29 \pm 0.82$ & $3.38 \pm 0.75$ & $3.14 \pm 0.94$ & 0.361 \\
\hline FVC \% predicted & $81.76 \pm 14.64$ & $83.23 \pm 14.24$ & $79.20 \pm 15.48$ & 0.402 \\
\hline $\mathrm{FEV}_{\mathrm{l}} / \mathrm{FVC}(\%)$ & $41.61 \pm 13.00$ & $38.89 \pm 9.80$ & $46.32 \pm 16.54$ & 0.128 \\
\hline FEF 25-75 (L) & $0.46 \pm 0.24$ & $0.43 \pm 0.19$ & $0.53 \pm 0.29$ & 0.224 \\
\hline FEF $25-75 \%$ predicted & $19.88 \pm 10.53$ & $18.04 \pm 8.69$ & $23.07 \pm 12.84$ & 0.191 \\
\hline Pre 6MWT (m) & $4|3.63 \pm 89.7|$ & $400.35 \pm 97.31$ & $436.67 \pm 72.03$ & 0.216 \\
\hline
\end{tabular}

Notes: Between-group differences were analyzed using the chi-square test, Fisher's exact test, or independent two-sample $t$-test. *Variables were analyzed using Fisher's exact test.

Abbreviations: 6MWT, 6-min walking test; BMI, body mass index; BODE index, the body mass index, airflow obstruction, dyspnea, and exercise capacity index; DM, diabetes mellitus; HTN, hypertension; CAT, chronic obstructive pulmonary disease assessment test; mMRC, modified Medical Research Council; FEF 25-75, forced expiratory flow from 25 to $75 \%$ of the vital capacity; FEV , forced expiratory volume in I s; FVC, forced vital capacity.

the BODE index. There was no firm evidence regarding minimal clinically important differences (MCID) for mMRC and BODE index in COPD patients. Although more than $100 \mathrm{~mL}$ in FEV1 and $26 \mathrm{~m}$ in $6 \mathrm{MWT}$ are regarded as MCID, both outcomes did not reach these values. ${ }^{30} \mathrm{At}$ the baseline study, both groups presented more than $400 \mathrm{~m}$ in 6MWT, which were relatively high compared with other studies. ${ }^{31,32}$ A possible ceiling effect might lead to insignificant changes in a 6MWTs.

PR is considered as one of the highly effective treatment interventions for patients with COPD, with reports stating that PR alleviates dyspnea and fatigue and improve physical and emotional functions. ${ }^{12}$ In particular, improved physical function is directly associated with 
Table 2 Changes in Clinical, Spirometry, and 6-Min Walking Test Outcomes from Baseline to 8 Weeks in Both Groups

\begin{tabular}{|l|l|l|l|}
\hline & $\begin{array}{l}\text { Compliant } \\
\text { Group } \\
(\mathbf{N}=\mathbf{2 6})\end{array}$ & $\begin{array}{l}\text { Noncompliant } \\
\text { Group } \\
\mathbf{( N = 1 5 )}\end{array}$ & P value \\
\hline CAT & $-4.62 \pm 4.61$ & $2.40 \pm 6.73$ & 0.002 \\
\hline mMRC & $-0.73 \pm 0.83$ & $-0.27 \pm 0.88$ & 0.183 \\
\hline $\begin{array}{l}\text { BODE } \\
\text { index }\end{array}$ & $-1.00 \pm 1.06$ & $-0.20 \pm 0.56$ & 0.010 \\
\hline FEV (L) & $\begin{array}{l}0.05 \pm 0.19 \\
(5.50 \pm 16.48 \%)^{*}\end{array}$ & $\begin{array}{l}-0.09 \pm 0.16 \\
(-4.65 \pm 10.65 \%)^{*}\end{array}$ & $\begin{array}{l}0.019 \\
(0.039)^{*}\end{array}$ \\
\hline $\begin{array}{l}\text { 6MWT } \\
(\mathrm{m})\end{array}$ & $\begin{array}{l}18.23 \pm 43.96 \\
(6.61 \pm 13.79 \%)^{*}\end{array}$ & $\begin{array}{l}4.87 \pm 46.25 \\
(3.22 \pm 17.09 \%)^{*}\end{array}$ & $\begin{array}{l}0.363 \\
(0.492)^{*}\end{array}$ \\
\hline
\end{tabular}

Notes: Between-group differences were analyzed using the independent twosample $t$-test. ${ }^{*}$ Changed ratio as a reference for the baseline value.

Abbreviations: 6MWT, 6-min walking test; BODE index, the body mass index, airflow obstruction, dyspnea, and exercise capacity index; CAT, chronic obstructive pulmonary disease assessment test; mMRC, modified Medical Research Council; $\mathrm{FEV}_{\mathrm{l}}$, forced expiratory volume in I s.

improved prognosis in patients with COPD. ${ }^{33}$ However, in real-world practice, PR is underperformed among these patients. $^{34,35}$ The standard PR protocol requires multiple visits, high medical costs, and specialists from various fields. In addition, adherence to prescribed PR remains relatively low. ${ }^{15}$ Therefore, a large proportion of patients with COPD do not benefit fully from an adequate

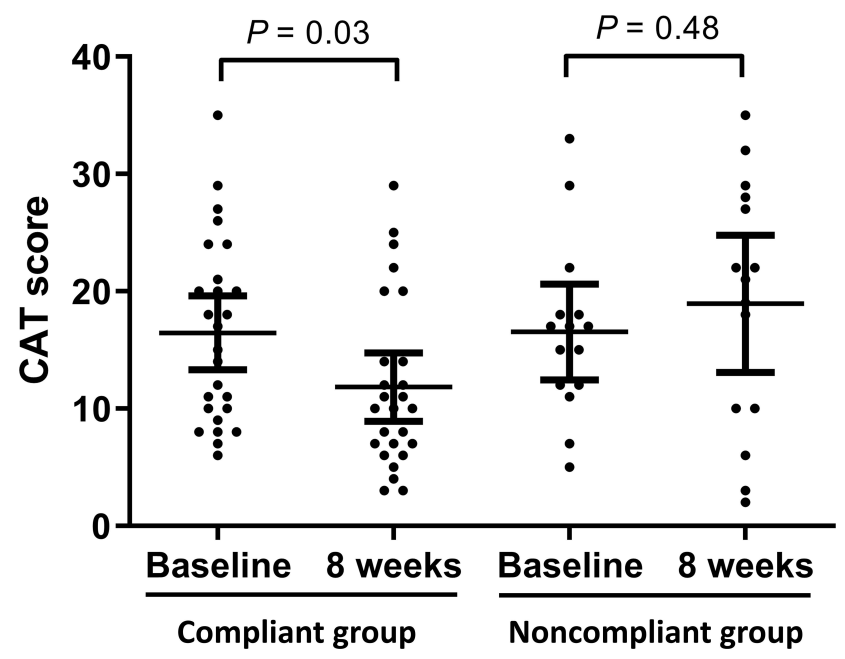

Figure 3 Primary outcome and CAT changes after 8 weeks of home-based pulmonary rehabilitation. A scatter plot representing chronic obstructive pulmonary disease (COPD) assessment test (CAT) results at baseline and 8 weeks. The plot is presented as mean with $95 \%$ confidence interval $(\mathrm{Cl})$. The differences between baseline values and values obtained after 8 weeks were analyzed using an independent two-sample $t$-test. In the compliant group, CAT scores significantly improved $(16.46 \pm 7.80$ vs $11.85 \pm 7.23 ; P=0.03)$, whereas in the noncompliant group, there was no significant change in CAT scores (16.53 \pm 7.38 vs $18.93 \pm$ 10.59; $P=0.48$ ). prescription for PR. To overcome this issue, HBPR might be applied, which is considered an alternative for standard PR. Nevertheless, the majority of studies on HBPR have included specialist supervision for PR, ${ }^{16-20}$ which might be another hurdle as it increases medical costs and the requirement of resources. ${ }^{20}$ In those studies, specialists periodically visited patients and reeducated them about inhaler use and training methods, supervised the exercise sessions, and encouraged the patients' usage of HBPR. Low socioeconomic status and transport-related inconveniences have been reported to be other reasons for the low adherence to PR. ${ }^{36-38}$ From this perspective, this study was meaningful as it demonstrated that unsupervised HBPR with one training session could still have beneficial effects on patients with COPD. This study could serve as the basis for a better treatment strategy for patients with COPD who have difficulties with frequent hospital visits.

Horton et $\mathrm{al}^{21}$ compared the effects of unsupervised HBPR with those of center-based PR. They found that HBPR failed to show noninferiority in most of the outcomes because several participants in the HBPR group did not complete the PR programs. Therefore, we hypothesized that compliance is a critical factor in the success of HBPR. In this study, the compliant patient group was compared with the noncompliant group. A recent retrospective study reported improved outcomes in compliant patients with unsupervised HBPR, although there were no significant differences, compared with the noncompliant patients. ${ }^{39}$ In this study, the results indeed demonstrated that the benefit of PR was noticeable only in the compliant group and that even one-time intervention could benefit the patients if they followed the training provided to them. Therefore, compliance, motivation, and appropriate education might be more important than where or how frequently these patients receive training in PR. Furthermore, the majority of participants preferred HBPR than frequent hospital visits for PR. ${ }^{21,40}$ In this context, the critical measure is educating patients about performing PR outside of the hospital.

There are several limitations to this study. First, a relatively small number of patients were included, and several exclusion criteria were applied to this study. For these reasons, the results could not be generalized to a broad COPD population, including long-term oxygen therapy users. Therefore, studies with larger sample sizes in a general COPD population would be needed to validate this approach. Second, the efficacy of unsupervised HBPR was assessed via a comparison between compliance and 

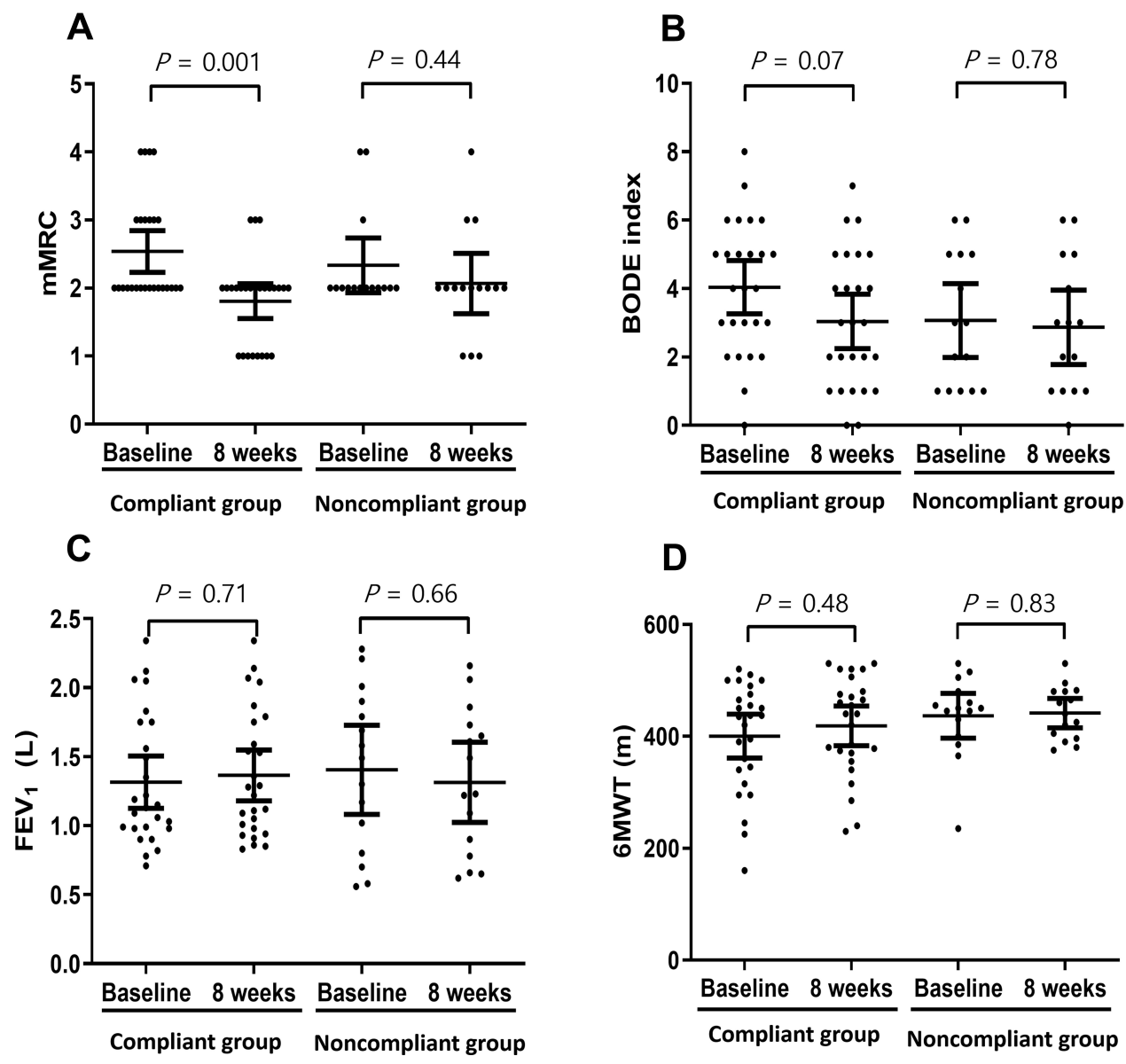

Figure 4 Secondary outcome measurements after 8 weeks of home-based pulmonary rehabilitation obtained for mMRC, BODE index, FEV presents baseline and 8 weeks outcomes. The plots are presented as mean with $95 \%$ confidence interval. Differences between the baseline values and values obtained after 8 weeks were analyzed using an independent two-sample $t$-test. In the compliant group, mMRC scores $(2.54 \pm 0.76 \mathrm{vs} \mathrm{I.8I} \pm 0.63 ; P=0.00 \mathrm{I},(\mathbf{A}))$ significantly improved after 8 weeks. However, the BODE index (4.04 \pm 1.93 vs $3.04 \pm 1.97 ; P=0.07$, (B)), FEVI (I.32 \pm 0.47 vs I.37 $\pm 0.46 ; P=0.7 \mathrm{I},(\mathbf{C}))$, and $6 \mathrm{MWT}$ results $(400.35 \pm 97.3 \mathrm{I}$ vs $4 \mathrm{I} 8.58 \pm$ 88.07; $P=0.48$, (D)) did not show significant changes. In the noncompliant group, mMRC scores ( $2.33 \pm 0.72$ vs $2.07 \pm 0.80 ; P=0.44,(\mathbf{A})$ ), BODE index (3.07 \pm 1.94 vs 2.87 $\pm 1.96 ; P=0.78$, (B)), FEVI $(I .4 \mathrm{I} \pm 0.58$ vs $\mathrm{I} .3 \mathrm{I} \pm 0.52 ; P=0.66,(C))$, and $6 \mathrm{MWT}$ results $(436.67 \pm 72.03$ vs $44 \mathrm{I} .53 \pm 47.20 ; P=0.83$, $(\mathrm{D}))$ were not significantly different. Abbreviations: $6 \mathrm{MWT}$, 6-min walking test; BODE index, the body mass index, airflow obstruction, dyspnea, and exercise capacity index; FEV ${ }_{1}$, forced expiratory volume in I s; mMRC, modified Medical Research Council.

noncompliance groups. However, we did not compare the education at 8-week intervals with standard PR. Besides, we observed the participants for only 8 weeks; possibly, a prolonged period would have yielded much better outcomes. Therefore, further study would be required to validate the noninferiority and the long-term effects of patient education at 8-week intervals for unsupervised HBPR. Finally, we investigated comorbidities depending on patients' questionnaires and electronic medical records. For this reason, we could not confirm whether these favorable outcomes were related to unsupervised HBPR for patients with COPD or with other comorbidities.

Despite these limitations, this study has indicated the importance of clinicians' efforts to promote PR in patients with COPD. These efforts are likely to lead to improved symptoms and quality of life in compliant patients.
Education about unsupervised HBPR could be conducted even when there is a lack of medical resources. Although a randomized controlled study with a large number of patients would be ideal, the present study demonstrates unsupervised HBPR as a possibility when it is not easy to prescribe standard PR for patients with COPD. Since only $\sim 60 \%$ of patients adhered to the unsupervised HBPR, further study on this strategy to promote compliance is necessary to benefit more patients with COPD.

Unsupervised HBPR performed for 8 weeks improved the health status in patients with COPD. These results suggest that compliant patients with COPD are likely to benefit from PR even if they could not visit hospitals as outpatients regularly, and when medical resources are lacking. Therefore, clinicians should recommend HBPR actively and make the necessary efforts to increase patients' compliance. 


\section{Abbreviations}

6MWT, the 6-min walking test; BODE index, the body mass index, airflow obstruction, dyspnea, and exercise capacity index; CAT, chronic obstructive pulmonary disease assessment test; COPD, chronic obstructive pulmonary disease; FEV1, forced expiratory volume in $1 \mathrm{~s}$; FVC, forced vital capacity; HBPR, home-based pulmonary rehabilitation; mMRC dyspnea scale, modified Medical Research Council dyspnea scale; PR, pulmonary rehabilitation.

\section{Data Sharing Statement}

All data relevant to the study can be acquired from the corresponding author by email. All data provided are deidentified to respect the privacy of patients who have participated in the trial.

\section{Funding}

This study was supported by grants (2018-0964) from the Asan Institute for Life Sciences, Asan Medical Center, Seoul, Korea.

\section{Disclosure}

The authors declare that they have no competing interests for this work.

\section{References}

1. Singh D, Agusti A, Anzueto A, et al. Global strategy for the diagnosis, management, and prevention of chronic obstructive lung disease: the GOLD science committee report 2019. Eur Respir J. 2019;53 (5):1900164. doi:10.1183/13993003.00164-2019

2. Calverley PMA, Anzueto AR, Carter K, et al. Tiotropium and olodaterol in the prevention of chronic obstructive pulmonary disease exacerbations (DYNAGITO): a double-blind, randomised, parallel-group, active-controlled trial. Lancet Respir Med. 2018;6 (5):337-344. doi:10.1016/S2213-2600(18)30102-4

3. Lipson DA, Barnhart F, Brealey N, et al. Once-daily single-inhaler triple versus dual therapy in patients with COPD. $N$ Engl $J$ Med. 2018;378(18):1671-1680. doi:10.1056/NEJMoa1713901

4. Wedzicha JA, Banerji D, Chapman KR, et al. Indacaterolglycopyrronium versus salmeterol-fluticasone for COPD. $N$ Engl $J$ Med. 2016;374(23):2222-2234. doi:10.1056/NEJMoa1516385

5. Rabe KF, Watz H. Chronic obstructive pulmonary disease. Lancet. 2017;389(10082):1931-1940. doi:10.1016/S0140-6736(17)31222-9

6. Spruit MA. Pulmonary rehabilitation. Eur Respir Rev. 2014;23 (131):55-63. doi:10.1183/09059180.00008013

7. Paz-Diaz H, Montes de Oca M, Lopez JM, Celli BR. Pulmonary rehabilitation improves depression, anxiety, dyspnea and health status in patients with COPD. Am J Phys Med Rehabil. 2007;86(1):30-36. doi:10.1097/PHM.0b013e31802b8eca

8. Moore E, Palmer T, Newson R, Majeed A, Quint JK, Soljak MA. Pulmonary rehabilitation as a mechanism to reduce hospitalizations for acute exacerbations of COPD: a systematic review and meta-analysis. Chest. 2016;150(4):837-859. doi:10.1016/j.chest.2016.05.038
9. Corhay JL, Dang DN, Van Cauwenberge H, Louis R. Pulmonary rehabilitation and COPD: providing patients a good environment for optimizing therapy. Int J Chron Obstruct Pulmon Dis. 2014;9:27-39.

10. Puhan MA, Scharplatz M, Troosters T, Steurer J. Respiratory rehabilitation after acute exacerbation of COPD may reduce risk for readmission and mortality - a systematic review. Respir Res. 2005;6:54. doi:10.1186/1465-9921-6-54

11. Garcia-Aymerich J, Lange P, Benet M, Schnohr P, Anto JM. Regular physical activity reduces hospital admission and mortality in chronic obstructive pulmonary disease: a population based cohort study. Thorax. 2006;61(9):772-778. doi:10.1136/thx.2006.060145

12. McCarthy B, Casey D, Devane D, Murphy K, Murphy E, Lacasse Y. Pulmonary rehabilitation for chronic obstructive pulmonary disease. Cochrane Database Syst Rev. 2015. doi:10.1002/14651858. CD003793.pub3(2).

13. Spruit MA, Singh SJ, Garvey C, et al. An official American Thoracic Society/European Respiratory Society statement: key concepts and advances in pulmonary rehabilitation. Am J Respir Crit Care Med. 2013;188(8):e13-64. doi:10.1164/rccm.201309-1634ST

14. Bolton CE, Bevan-Smith EF, Blakey JD, et al. British Thoracic Society guideline on pulmonary rehabilitation in adults. Thorax. 2013;68(Suppl 2):ii1-30. doi:10.1136/thoraxjnl-2013-203808

15. Young J, Jordan RE, Adab P, Enocson A, Jolly K. Interventions to promote referral, uptake and adherence to pulmonary rehabilitation for people with chronic obstructive pulmonary disease (COPD). Cochrane Database Syst Rev. 2017. doi:10.1002/14651858.CD012813(10).

16. Maltais F, Bourbeau J, Shapiro S, et al. Effects of home-based pulmonary rehabilitation in patients with chronic obstructive pulmonary disease: a randomized trial. Ann Intern Med. 2008;149 (12):869-878. doi:10.7326/0003-4819-149-12-200812160-00006

17. Vieira DS, Maltais F, Bourbeau J. Home-based pulmonary rehabilitation in chronic obstructive pulmonary disease patients. Curr Opin Pulm Med. 2010;16(2):134-143. doi:10.1097/MCP.0b013e32833642f2

18. Dias FD, Sampaio LM, da Silva GA, et al. Home-based pulmonary rehabilitation in patients with chronic obstructive pulmonary disease: a randomized clinical trial. Int J Chron Obstruct Pulmon Dis. 2013;8:537-544. doi:10.2147/COPD.S50213

19. Ninot G, Moullec G, Picot MC, et al. Cost-saving effect of supervised exercise associated to COPD self-management education program. Respir Med. 2011;105(3):377-385. doi:10.1016/j. rmed.2010.10.002

20. Holland AE, Mahal A, Hill CJ, et al. Home-based rehabilitation for COPD using minimal resources: a randomised, controlled equivalence trial. Thorax. 2017;72(1):57-65.

21. Horton EJ, Mitchell KE, Johnson-Warrington V, et al. Comparison of a structured home-based rehabilitation programme with conventional supervised pulmonary rehabilitation: a randomised noninferiority trial. Thorax. 2018;73(1):29-36. doi:10.1136/thoraxjnl-2016-208506

22. Jones PW, Harding G, Berry P, Wiklund I, Chen WH, Kline Leidy N. Development and first validation of the COPD assessment test. Eur Respir J. 2009;34(3):648-654. doi:10.1183/09031936.00102509

23. Zainuldin MR, Knoke D, Mackey MG, Luxton N, Alison JA. Prescribing cycle training intensity from the six-minute walk test for patients with COPD. BMC Pulm Med. 2007;7:9. doi:10.1186/ 1471-2466-7-9

24. Zainuldin R, Mackey MG, Alison JA. Prescription of walking exercise intensity from the 6-minute walk test in people with chronic obstructive pulmonary disease. J Cardiopulm Rehabil Prev. 2015;35 (1):65-69. doi:10.1097/HCR.0000000000000074

25. Mahler DA, Horowitz MB. Perception of breathlessness during exercise in patients with respiratory disease. Med Sci Sports Exerc. 1994;26(9):1078-1081. doi:10.1249/00005768-199409000-00002

26. Celli BR, Cote CG, Marin JM, et al. The body-mass index, airflow obstruction, dyspnea, and exercise capacity index in chronic obstructive pulmonary disease. $N$ Engl J Med. 2004;350(10):1005-1012. doi:10.1056/NEJMoa021322 
27. Kon SS, Canavan JL, Jones SE, et al. Minimum clinically important difference for the COPD assessment test: a prospective analysis. Lance Respir Med. 2014;2(3):195-203. doi:10.1016/S2213-2600(14)70001-3

28. Ghobadi H, Ahari SS, Kameli A, Lari SM. The relationship between COPD assessment test (CAT) scores and severity of airflow obstruction in stable COPD patients. Tanaffos. 2012;11(2):22-26.

29. Cote CG, Celli BR. Pulmonary rehabilitation and the BODE index in COPD. Eur Respir J. 2005;26(4):630-636. doi:10.1183/ 09031936.05.00045505

30. Jones PW, Beeh KM, Chapman KR, Decramer M, Mahler DA, Wedzicha JA. Minimal clinically important differences in pharmacological trials. Am J Respir Crit Care Med. 2014;189(3):250-255. doi:10.1164/rccm.201310-1863PP

31. Celli B, Tetzlaff K, Criner G, et al. The 6-minute-walk distance test as a chronic obstructive pulmonary disease stratification tool. Insights from the COPD biomarker qualification consortium. Am J Respir Crit Care Med. 2016;194(12):1483-1493. doi:10.1164/rccm.201508-1653OC

32. Dajczman E, Wardini R, Kasymjanova G, Préfontaine D, Baltzan MA, Wolkove N. Six minute walk distance is a predictor of survival in patients with chronic obstructive pulmonary disease undergoing pulmonary rehabilitation. Can Respir J. 2015;22 (4):225-229. doi:10.1155/2015/280187

33. Waschki B, Kirsten A, Holz O, et al. Physical activity is the strongest predictor of all-cause mortality in patients with COPD: a prospective cohort study. Chest. 2011;140(2):331-342. doi:10.1378/chest.10-2521

34. Desveaux L, Janaudis-Ferreira T, Goldstein R, Brooks D. An international comparison of pulmonary rehabilitation: a systematic review. COPD. 2015;12(2):144-153. doi:10.3109/15412555.2014.922066
35. Guo SE, Bruce A, Chalmers JD. Improving understanding of and adherence to pulmonary rehabilitation in patients with COPD: a qualitative inquiry of patient and health professional perspectives. PLoS One. 2014;9(10):e110835. doi:10.1371/journal.pone.0110835

36. Oates GR, Hamby BW, Stepanikova I, et al. Social determinants of adherence to pulmonary rehabilitation for chronic obstructive pulmonary disease. Copd. 2017;14(6):610-617. doi:10.1080/15412555. 2017.1379070

37. Keating A, Lee AL, Holland AE. Lack of perceived benefit and inadequate transport influence uptake and completion of pulmonary rehabilitation in people with chronic obstructive pulmonary disease: a qualitative study. J Physiother. 2011;57(3):183-190. doi:10.1016/ S1836-9553(11)70040-6

38. Burge AT, Holland AE, McDonald CF, et al. Home-based pulmonary rehabilitation for COPD using minimal resources: an economic analysis. Respirology. 2019. doi:10.1111/resp.13667.

39. Candemir I, Ergun P, Kaymaz D, Demir N, McCurdy SA. Comparison of unsupervised home-based pulmonary rehabilitation versus supervised hospital outpatient pulmonary rehabilitation in patients with chronic obstructive pulmonary disease. Expert Rev Respir Med. 2019;13 (12):1195-1203. doi:10.1080/17476348.2019.1675516

40. Lahham A, McDonald CF, Mahal A, et al. Home-based pulmonary rehabilitation for people with COPD: a qualitative study reporting the patient perspective. Chron Respir Dis. 2018;15(2):123-130. doi:10.1177/1479972317729050

\section{Publish your work in this journal}

The International Journal of COPD is an international, peer-reviewed journal of therapeutics and pharmacology focusing on concise rapid reporting of clinical studies and reviews in COPD. Special focus is given to the pathophysiological processes underlying the disease, intervention programs, patient focused education, and self managemen protocols. This journal is indexed on PubMed Central, MedLine and CAS. The manuscript management system is completely online and includes a very quick and fair peer-review system, which is all easy to use. Visit http://www.dovepress.com/testimonials.php to read real quotes from published authors. 